\title{
An intrinsic Liouville theorem for degenerate parabolic equations
}

\author{
Eduardo V. Teixeira \& José Miguel Urbano
}

May 29, 2014

\begin{abstract}
We show that weak solutions of the degenerate $p$-Laplace equation

$$
u_{t}-\operatorname{div}\left(|\nabla u|^{p-2} \nabla u\right)=0, \quad p \geq 2
$$

in the whole space are constant if their growth at infinity is properly controlled in an intrinsic manner.
\end{abstract}

Keywords: Degenerate parabolic equations; Liouville theorem; intrinsic scaling. 2010 Mathematics Subject Classification. 35K55, 35K65, 35B53.

\section{Introduction}

A classical result from Complex Analysis asserts that if $h: \mathbb{C} \rightarrow \mathbb{C}$ is an entire, bounded holomorphic function then $h$ must be constant. This keystone result was originally proven by the french mathematician Joseph Liouville back in the 19th century [6]. Nowadays results of such a nature - usually called Liouville-type theorems - are spread all over the modern theory of mathematical analysis and applications.

Within the theory of elliptic PDEs, the most basic Liouville theorem asserts that bounded, harmonic functions, $\Delta h=0$, defined in $\mathbb{R}^{n}$ must be constant. There are quite a few different ways to prove this result. Probably the simplest form is by exploring the mean value property for harmonic functions, i.e., $h(y)=f_{B_{r}(y)} h(x) d x$ : since $h$ is bounded, the difference between the average in balls centered at two points is $o(1)$ as the radius tends to infinity. Despite its elegancy, the above argument is confined to the Laplace equation. Moser, in his fundamental work [9], noticed that a Liouville-type theorem could be derived by means of Harnack's inequality. In particular, bounded entire solutions to $\operatorname{div}\left(a_{i j}(x) \nabla u\right)=0$ must be constant, provided $a_{i j}$ is uniformly elliptic.

As a matter of fact, Moser's argument for proving a Liouville-type theorem only requires a one-sided bound on the solution. For evolutionary problems though, a one side bound is not enough to conclude that solutions in $\mathbb{R}^{n} \times \mathbb{R}$ have to be constant. For example, $u(x, t)=e^{x+t}$ is a non-constant solution to the heat equation $u_{t}-u^{\prime \prime}=0$ in $\mathbb{R} \times \mathbb{R}$. Liouville's theorem, 
as stated above, also fails to hold if the equation is non-homogeneous. This is easily verified: the function $x \mapsto|x|^{2}$, for instance, is not constant and satisfies $\Delta\left(|x|^{2}\right)=2$ in $\mathbb{R}^{n}$.

Bernstein, in his study about global minimal surfaces, established that any entire solution to a linear, uniformly elliptic equation in the plane,

$$
a_{11}(x, y) u_{x x}+2 a_{12}(x, y) u_{x y}+a_{22}(x, y) u_{y y}=0, \quad \text { in } \mathbb{R}^{2},
$$

which grows sublinearly at infinity, must be constant. The proof of Bernstein's theorem was revisited in [3] and [7], and since then a number of other proofs were derived, see [8] for a particularly striking one. The result can be seen as a refinement of Liouville's Theorem - a solution to a uniformly elliptic equations in the plane that grows as $\mathrm{o}(|x|)$ at infinity must be constant.

The goal of this note is to obtain a Bernstein result for degenerate parabolic equations of the form

$$
u_{t}-\operatorname{div}\left(|\nabla u|^{p-2} \nabla u\right)=0 \quad \text { in } \mathbb{R}^{n} \times \mathbb{R},
$$

where $p>2$. This class of equations has been thoroughly studied in the last 30 years and is now quite well understood (see the standard reference [1] and the more recent account in [13]). The approach of [12], where a precise and sharp derivation of the Hölder exponent of solutions is carried out, shifted the focus of the analysis from quantitative to qualitative results (see also, in this direction, [4] and [5]). We proceed here in this vein, showing that solutions of (1.1) are forced to remain constant if their growth at infinity is controlled. We will show that the rate of decay is given by a power of the appropriate intrinsic norm, explored in $[12]$.

Probabilistic interpretations of Liouville's theorem (e.g. [10]) are quite helpful for an intuitive understanding of the theorem we shall present here. Our proof though is based on the geometric tangential analysis recently developed in [12], combined with an intrinsic blow-up argument.

\section{An intrinsic Liouville theorem}

We consider local weak solutions (for the precise definition see [12])

$$
u \in C_{\mathrm{loc}}\left(\mathbb{R} ; L_{\mathrm{loc}}^{2}\left(\mathbb{R}^{n}\right)\right) \cap L_{\mathrm{loc}}^{p}\left(\mathbb{R} ; W_{\mathrm{loc}}^{1, p}\left(\mathbb{R}^{n}\right)\right)
$$

of the degenerate parabolic equation

$$
u_{t}-\operatorname{div}\left(|\nabla u|^{p-2} \nabla u\right)=0, \quad p>2 .
$$

Given $0<\alpha<1$, define the $(2, p)$-interpolator

$$
\theta_{\alpha}:=2 \alpha+p(1-\alpha),
$$

which clearly satisfies $2<\theta_{\alpha}<p$. For such $\theta_{\alpha}$, consider the intrinsic parabolic cylinders

$$
G_{\tau}^{\theta_{\alpha}}:=\left(-\frac{1}{2} \tau^{\theta_{\alpha}}, \frac{1}{2} \tau^{\theta_{\alpha}}\right) \times B_{\tau}(0), \quad \tau>0
$$


and the intrinsic norm of a point $(x, t)$

$$
\|(x, t)\|_{\theta_{\alpha}}:=|t|^{1 / \theta_{\alpha}}+|x| .
$$

Theorem 1. Let $u$ be an entire solution to

$$
u_{t}-\operatorname{div}\left(|\nabla u|^{p-2} \nabla u\right)=0, \quad p>2 .
$$

Suppose, for some $0<\alpha<1$, there holds

$$
u(x, t)=O\left(\|(x, t)\|_{\theta_{\alpha}}^{\alpha}\right),
$$

as $\|(x, t)\|_{\theta_{\alpha}} \rightarrow \infty$. Then $u$ is constant.

Proof. Without loss of generality, we assume $u(0,0)=0$. Fix a large positive number $\ell \gg 1$ and define in $G_{1}^{\theta_{\alpha}} \equiv G_{1}$ the intrinsically scaled function

$$
V_{\ell}(x, t):=\frac{u\left(\ell x, \ell^{\theta_{\alpha}} t\right)}{\ell^{\alpha}} .
$$

We first show that

$$
\sup _{G_{1}}\left|V_{\ell}\right| \leq C,
$$

for $\ell \gg 1$. Let $\left(x_{\ell}, t_{\ell}\right)$ be a point in $\overline{G_{1}}$ such that

$$
\sup _{G_{1}}\left|V_{\ell}\right|=\left|V_{\ell}\left(x_{\ell}, t_{\ell}\right)\right|=\left|\frac{u\left(\ell x_{\ell}, \ell^{\theta_{\alpha}} t_{\ell}\right)}{\ell^{\alpha}}\right| .
$$

We have

$$
\left\|\left(\ell x_{\ell}, \ell^{\theta_{\alpha}} t_{\ell}\right)\right\|_{\theta_{\alpha}}^{\alpha}=\left(\left|\ell^{\theta_{\alpha}} t_{\ell}\right|^{1 / \theta_{\alpha}}+\left|\ell x_{\ell}\right|\right)^{\alpha} \leq(2 \ell)^{\alpha},
$$

hence

$$
\frac{1}{2^{\alpha}} \sup _{G_{1}}\left|V_{\ell}\right|=\left|\frac{u\left(\ell x_{\ell}, \ell^{\theta_{\alpha}} t_{\ell}\right)}{(2 \ell)^{\alpha}}\right| \leq \frac{\left|u\left(\ell x_{\ell}, \ell^{\theta_{\alpha}} t_{\ell}\right)\right|}{\left\|\left(\ell x_{\ell}, \ell^{\theta_{\alpha}} t_{\ell}\right)\right\|_{\theta_{\alpha}}^{\alpha}} .
$$

We now take the limit as $\ell \rightarrow \infty$. If $\left\|\left(\ell x_{\ell}, \ell^{\theta_{\alpha}} t_{\ell}\right)\right\|_{\theta_{\alpha}} \rightarrow \infty$, the right-hand side in (2.6) is bounded, due to condition (2.4), and (2.5) holds. If, on the contrary, $\left\|\left(\ell x_{\ell}, \ell^{\theta_{\alpha}} t_{\ell}\right)\right\|_{\theta_{\alpha}}$ remains bounded then, since $u$ is continuous,

$$
\left|\frac{u\left(\ell x_{\ell}, \ell^{\theta_{\alpha}} t_{\ell}\right)}{(2 \ell)^{\alpha}}\right| \leq \frac{C}{(2 \ell)^{\alpha}} \longrightarrow 0
$$

and (2.5) still holds.

We now follow [12] and compute

$$
\partial_{t} V_{\ell}(x, t)=\ell^{\theta_{\alpha}-\alpha} u_{t}\left(\ell x, \ell^{\theta_{\alpha}} t\right)
$$

and

$$
\operatorname{div}\left(\left|\nabla V_{\ell}(x, t)\right|^{p-2} \nabla V_{\ell}(x, t)\right)=\ell^{p-(p-1) \alpha} \operatorname{div}\left(\left|\nabla u\left(\ell x, \ell^{\theta_{\alpha}} t\right)\right|^{p-2} \nabla u\left(\ell x, \ell^{\theta_{\alpha}} t\right)\right)
$$


to conclude, recalling $(2.2)$, that

$$
\partial_{t} V_{\ell}-\operatorname{div}\left(\left|\nabla V_{\ell}\right|^{p-2} \nabla V_{\ell}\right)=0
$$

in $G_{1}$.

Next, fix $\alpha<\beta<1$ and the corresponding $\theta_{\beta}$. Notice, from (2.2), that $\theta_{\alpha}$ is a decreasing function of $\alpha$; hence $\theta_{\beta}<\theta_{\alpha}$. From [12, Theorem 3.4], we obtain

$$
\left|V_{\ell}(x, t)\right| \leq C\left(|t|^{1 / \theta_{\beta}}+|x|\right)^{\beta}, \quad \forall(x, t) \in G_{1 / 2}^{\theta_{\beta}}
$$

where $C$ is universal. Then, after scaling,

$$
\begin{aligned}
\sup _{G_{\ell / 2}^{\theta_{\beta}}} \frac{|u(x, t)|}{\left(|t|^{1 / \theta_{\beta}}+|x|\right)^{\beta}} & =\sup _{G_{1 / 2}^{\theta_{\beta}}} \frac{\left|u\left(\ell x, \ell^{\theta_{\beta}} t\right)\right|}{\ell^{\beta}\left(|t|^{1 / \theta_{\beta}}+|x|\right)^{\beta}} \\
& =\ell^{\alpha-\beta} \sup _{G_{1 / 2}^{\theta_{\beta}}} \frac{\left|V_{\ell}\left(x, \ell^{-\theta_{\alpha}+\theta_{\beta}} t\right)\right|}{\left(|t|^{1 / \theta_{\beta}}+|x|\right)^{\beta}} \\
& \leq \ell^{\alpha-\beta} \sup _{G_{1 / 2}^{\theta_{\beta}}} \frac{\left|V_{\ell}\left(x, \ell^{-\theta_{\alpha}+\theta_{\beta}} t\right)\right|}{\left(\left|\ell^{-\theta_{\alpha}+\theta_{\beta}} \cdot t\right|^{1 / \theta_{\beta}}+|x|\right)^{\beta}} \\
& =\mathrm{o}(1),
\end{aligned}
$$

as $\ell \rightarrow \infty$, by virtue of estimate (2.8). Observe that, taking $\ell \gg 1$ large enough, we can assume $\ell^{-\theta_{\alpha}+\theta_{\beta}}<1$. Notice also that $\left(x, \ell^{-\theta_{\alpha}+\theta_{\beta}} t\right)$ does belong to $G_{1 / 2}^{\theta_{\beta}}$ provided $(x, t)$ does.

Clearly (2.9) implies that $u$ is zero in the whole $\mathbb{R}^{n} \times \mathbb{R}$ and the proof of the theorem is concluded.

Remark. Theorem 1 is a generalization of [2, Theorem 1.1], at least for the case of the $p$-Laplacian and assuming $T=+\infty$. To the best of our knowledge, the result is new even in the elliptic case.

Acknowledgements. This work was developed in the framework of the Brazilian Program "Ciência sem Fronteiras". Research of EVT partially supported by CNPq-Brazil. Research of JMU supported by projects UTA-CMU/MAT/0007/2009 and PTDC/MAT-CAL/0749/2012 and by CMUC, funded by the European Regional Development Fund through the program COMPETE and by FCT, under the project PEst-C/MAT/UI0324/2011.

\section{References}

[1] E. DiBenedetto, Degenerate Parabolic Equations, Springer-Verlag, New York, 1993.

[2] E. DiBenedetto, U. Gianazza and V. Vespri, Liouville-type theorems for certain degenerate and singular parabolic equations, C. R. Math. Acad. Sci. Paris 348 (2010), 873-877. 
[3] E. Hopf, On S. Bernstein's theorem on surfaces z(x,y) of nonpositive curvature, Proc. Amer. Math. Soc. 1 (1950), 80-85.

[4] T. Iwaniec and J.J. Manfredi, Regularity of p-harmonic functions on the plane, Rev. Mat. Iberoamericana 5 (1989), 1-19.

[5] T. Kuusi, G. Mingione and K. Nystrom, Sharp regularity for evolutionary obstacle problems, interpolative geometries and removable sets, J. Math. Pures Appl., to appear.

[6] J. Liouville, Leçons sur les fonctions doublement périodiques, J. Reine Angew. Math. 89 (1880), 277-310.

[7] E. Mickle, A remark on a theorem of Serge Bernstein, Proc. Amer. Math. Soc. 1 (1950), $86-89$.

[8] J. Nitsche, Elementary proof of Bernstein's theorem on minimal surfaces, Ann. of Math. (2) 66 (1957), 543-544.

[9] J. Moser, On Harnack's theorem for elliptic differential equation, Comm. Pure Appl. Math. 14 (1961), 577-591.

[10] E. Priola and J. Zabczyk, Liouville theorems for non-local operators, J. Funct. Anal. 216 (2004), 455-490.

[11] E. V. Teixeira, Sharp regularity for general Poisson equations with borderline sources, J. Math. Pures Appl. 99 (2013), 150-164.

[12] E.V. Teixeira and J.M. Urbano, A geometric tangential approach to sharp regularity for degenerate evolution equations, Anal. PDE, to appear.

[13] J.M. Urbano, The Method of Intrinsic Scaling, Lecture Notes in Mathematics 1930, Springer-Verlag, Berlin, 2008.

EDUARDo V. TEIXEIRA

Universidade Federal do Ceará

Campus of Pici - Bloco 914

Fortaleza - Ceará - Brazil

60.455-760

teixeira@mat.ufc.br
José Miguel Urbano

CMUC, Department of Mathematics

University of Coimbra

3001-501 Coimbra

Portugal

jmurb@mat.uc.pt 